\title{
The Effects of Combined Exercise on Health-Related Fitness, Endotoxin, and Immune Function of Postmenopausal Women with Abdominal Obesity
}

\author{
Sung-Mo Park, Yi-Sub Kwak, and Jin-Goo Ji \\ Department of Physical Education, Dong-Eui University, 995 Eomgwangno, Busanjin-gu, Busan 614-714, Republic of Korea \\ Correspondence should be addressed to Jin-Goo Ji; jjg199@hanmail.net
}

Received 17 February 2015; Revised 27 April 2015; Accepted 28 April 2015

Academic Editor: Senthamil R. Selvan

Copyright (c) 2015 Sung-Mo Park et al. This is an open access article distributed under the Creative Commons Attribution License, which permits unrestricted use, distribution, and reproduction in any medium, provided the original work is properly cited.

\begin{abstract}
This study was conducted to examine the effects of combined exercise on health-related fitness, endotoxin concentrations, and immune functions of postmenopausal women with abdominal obesity. 20 voluntary participants were recruited and they were randomly allocated to the combined exercise group $(n=10)$ or the control group $(n=10)$. Visceral obesity was defined as a visceral-to-subcutaneous fat ratio $\geq 0.4$ based on computed tomography (CT) results. Body composition, exercise stress testing, fitness measurement, CT scan, and blood variables were analyzed to elucidate the effects of combined exercise. The SPSS Statistics 18.0 program was used to calculate means and standard deviations for all variables. Significant differences between the exercise group and control group were determined with 2-way ANOVA and paired $t$-tests. The exercise group's abdominal obesity was mitigated due to visceral fat reduction; grip strength, push-ups, and oxygen uptake per weight improved; and HDL-C and IgA level also increased, while TNF- $\alpha$, CD14, and endotoxin levels decreased. Lowered TNF- $\alpha$ after exercise might have an important role in the obesity reduction. Therefore, we can conclude that combined exercise is effective in mitigating abdominal obesity, preventing metabolic diseases, and enhancing immune function.
\end{abstract}

\section{Introduction}

In Korea, $26.5 \%$ of the total female population is obese, and women in their 30 s, 40 s, and 50 s account for $28 \%, 34.4 \%$, and $37.4 \%$, respectively, of the total obese female population. A sharp increase in the prevalence of obesity has been particularly seen with onset of menopause [1], a time in which women experience many metabolic changes from female hormone fluctuations. It has been reported that women are at higher risk for obesity during this period due to increases in body fat [2]. Postmenopausal women, in particular, often develop abdominal obesity without weight change, due to excessive accumulation of visceral fat and hormonal changes in the abdomen [3].

Increased physical activity through regular exercise is very effective for mitigating obesity and improving healthrelated fitness at all ages $[4,5]$. Park et al. [6] reported that combined exercise, resistance exercise combined with aerobic training, is more effective for mitigating obesity than aerobic training alone.

Recently, it was reported that gut microbiota is closely involved with obesity onset [7] and that endotoxins residing in gut flora are also closely correlated with visceral fat increases [8]. It was also reported that endotoxins suppress immune functions by inducing inflammatory responses [9].

Endotoxins promote the activation of inflammatory responses by stimulating microphages to secrete proinflammatory cytokines, including tumor necrosis factor- $\alpha$ (TNF$\alpha)$ [10]. During this process, endotoxins are induced to bind to CD14 on the microphage membrane [11]. The endotoxin receptor CD14 plays an important role in inflammatory and innate immune responses [12-14]. As obesity can increase endotoxin levels, causing inflammatory responses, obese women can in turn suppress immune function.

In this vein, various studies on controlling endotoxin levels and immune functions are being conducted, and interest 
in reducing endotoxins and improving immune functions through exercise is increasing $[15,16]$.

Kim et al. [17] reported that the largest immune function improvements resulted from combined exercise when aerobic, resistance, and combined exercises were compared. $\mathrm{Ng}$ et al. [18] reported that there were no changes in concentrations of serum endotoxin and TNF- $\alpha$ after a onetime $21 \mathrm{~km}$ road race. Sloan et al. [19] reported that when 61 study participants performed high- and moderate-intensity aerobic exercise for 30-40 min, 4 times a week for 12 weeks, only high-intensity exercise decreased the concentration of serum endotoxins.

Clearly, there have been many studies about an acute bout of exercise and aerobic exercise on physical activity and immune functions, but there is a lack of studies on endotoxin concentration changes after combined physical exercise. Moreover, while there are many studies on obesity and inflammation, as well as exercise and immune functions, there are hardly any studies on the interplay between endotoxins, health-related fitness, and immune functions of postmenopausal women with abdominal obesity.

Accordingly, this study was conducted to examine the effects of combined exercise on health-related fitness, endotoxin concentrations, and immune functions of postmenopausal women with abdominal obesity.

\section{Study Methods}

2.1. Participants. The participants in this study were naturally postmenopausal middle-aged women with abdominal obesity. Other selection criteria included a lack of regular exercise habits, no prior or present history of genital-related diseases, and not being under treatment for any internal or gynecological diseases. As we explained above, 20 voluntary participants were randomly allocated to the combined exercise group $(n=10)$ or the control group $(n=10)$. And this research was conducted ethically according to international standards.

Visceral obesity was defined as a visceral-to-subcutaneous fat ratio $\geq 0.4$ based on computed tomography (CT) results.

The participants' physical characteristics are summarized in Table 1.

\subsection{Test Methods}

2.2.1. Body Composition Examination. A body composition analyzer (VENUS-5.5, Korea) was used to measure the height, weight, \% body fat, body fat, and lean body mass (LBM) before and after 12 weeks of exercise. Body mass index (BMI) was calculated using weight/height $\left(\mathrm{kg} / \mathrm{m}^{2}\right)$. Blood pressure was measured with a mercury sphygmomanometer (Hico, Japan) after participants were stabilized for $30 \mathrm{~min}$.

2.2.2. Exercise Stress Testing. Exercise stress testing was conducted before and after 12 weeks of exercise, using the Balke treadmill protocol. Maximal exercise stress was defined as the presence of any 2 of the following: (1) manifestation of
TABLE 1: The characteristic subjects.

\begin{tabular}{lcc}
\hline Variable & $\begin{array}{c}\text { Exercise group } \\
(n=10)\end{array}$ & $\begin{array}{c}\text { Control group } \\
(n=10)\end{array}$ \\
\hline Age $(\mathrm{yr})$ & $57.20 \pm 2.57$ & $57.20 \pm 1.69$ \\
Body weight $(\mathrm{kg})$ & $64.90 \pm 3.83$ & $64.76 \pm 3.26$ \\
Height $(\mathrm{cm})$ & $157.95 \pm 2.57$ & $155.62 \pm 3.14$ \\
Percent body fat $(\%)$ & $34.32 \pm 2.79$ & $34.63 \pm 1.60$ \\
BMI $\left(\mathrm{kg} / \mathrm{m}^{2}\right)$ & $26.02 \pm 1.55$ & $26.80 \pm 1.09$ \\
\hline
\end{tabular}

BMI: body mass index.

steady state with oxygen intake not exceeding $150 \mathrm{~mL} / \mathrm{min}$ and expected maximum heart rate (220-age); (2) respiratory exchange ratio $(\mathrm{RER}) \geq 1.15$; and (3) ratings of perceived exertion $(\mathrm{RPE}) \geq 17$ [20]. Oxygen intake and heart rate during exercise were analyzed with an automatic metabolic analyzer (Quarkb2, Cosmed, Italy).

2.2.3. Combined Exercise Program. In the week prior to training, 1-repetition maximum (1RM) measurements were taken, and resistance equipment training and treadmill acclimation exercises were performed. The 1RM for resistance training was measured using the formula from the Korea Institute of Science and Technology [21]. In weeks $1-6,60 \%$ of $1 \mathrm{RM}$ was used for 8-10 repetitions. In weeks 7-12, 70\% 1RM was used for 10-12 repetitions. Each session lasted for 30 min 3 times a week. For aerobic training, exercise speed and heart rate based on the maximal exercise test were used to calculate the target heart rate (THR), equivalent to $40-75 \%$ of the heart rate reserve $(\mathrm{HRR}),(\mathrm{THR}=\mathrm{HRR} \times$ intensity $(\%)+$ resting heart rate), after which the target exercise speed was determined with a regression equation each for $40 \mathrm{~min} 3$ times a week for 12 weeks.

The exercise program is as shown in Table 2.

2.2.4. Fitness Measurement. Left and right grip strengths were measured with a hand dynamometer (T.K.K. 5101). Back strength was measured with a back dynamometer (T.K.K. 5102). And also, total sit-ups and push-ups performed in 30 seconds were measured. Flexibility was measured using the sit-and-reach test. All measurements were taken twice, and the highest value was recorded.

2.2.5. CT Scan. To measure abdominal fat, a CT scanner (Samsung GE Sytec 3000 I) scanned the transverse sections one above and one below umbilical level. The area's total abdominal fat volume was calculated by considering tissues with a Hounsfield number from -250 to -50 to be fat. Using abdominal and peritoneal spread as the boundary, the areas were divided into visceral fat tissue and subcutaneous fat tissue, with which the visceral fat to subcutaneous fat ratio was calculated.

2.2.6. Blood Analysis. Before and after program, $15 \mathrm{~mL}$ of blood was drawn from the antecubital vein under fasting, stable conditions. Levels of total cholesterol (TC), triglycerides, high-density lipoprotein cholesterol (HDL-C), 
TABLE 2: Combined exercise programs.

\begin{tabular}{|c|c|c|c|}
\hline Parameter & & Event & Time \\
\hline Warm-up & \multicolumn{2}{|c|}{ Head walk, neck stretch, shoulder circling, ribs extension, standing side raise, leg stretch, and triangle stretch } & $10 \mathrm{~min}$ \\
\hline \multirow{4}{*}{$\begin{array}{l}\text { Aerobic } \\
\text { exercise }\end{array}$} & Variety & Duration & \multirow{4}{*}{$40 \mathrm{~min}$} \\
\hline & \multirow{3}{*}{ Running } & 1 6 weeks HRR 40 55\% & \\
\hline & & 7 12 weeks HRR & \\
\hline & & $56 \sim 75 \%$ & \\
\hline \multirow{6}{*}{$\begin{array}{l}\text { Resistance } \\
\text { exercise }\end{array}$} & Variety & Duration & \multirow{6}{*}{$30 \mathrm{~min}$} \\
\hline & \multirow{5}{*}{$\begin{array}{l}\text { Arm curl, leg curl, leg extension, } \\
\text { squat, chest press, low back, } \\
\text { shoulder press, lat pulldown, } \\
\text { back extension, and sit-up }\end{array}$} & $1 \sim 6$ weeks & \\
\hline & & $1 \mathrm{RM} 60 \%$ & \\
\hline & & $8-10$ repetition $\times 3$ sets & \\
\hline & & 7 12 weeks & \\
\hline & & $1 \mathrm{RM} 70 \%$ & \\
\hline Cool-down & & Hand and leg shaking, stretch, and walking & $10 \mathrm{~min}$ \\
\hline
\end{tabular}

TABLE 3: The changes of abdominal fat between the groups at baseline and after 12 weeks of training.

\begin{tabular}{|c|c|c|c|c|}
\hline Variables & Group & Baseline & 12 weeks & $F$-value \\
\hline \multirow{2}{*}{ Total fat volume $\left(\mathrm{cm}^{3}\right)$} & Exercise & $741.61 \pm 90.98$ & $667.53 \pm 84.64$ & \multirow{2}{*}{$G \times P: 2.592$} \\
\hline & Control & $738.67 \pm 87.86$ & $753.41 \pm 85.29$ & \\
\hline \multirow{2}{*}{ Visceral fat volume $\left(\mathrm{cm}^{3}\right)$} & Exercise & $238.78 \pm 31.69$ & $198.27 \pm 27.97^{* *}$ & \multirow{2}{*}{$\mathrm{G} \times \mathrm{P}: 7.619^{\# \#}$} \\
\hline & Control & $237.00 \pm 30.90$ & $248.78 \pm 29.12$ & \\
\hline \multirow{2}{*}{ Subcutaneous fat volume $\left(\mathrm{cm}^{3}\right)$} & Exercise & $502.84 \pm 60.66$ & $469.26 \pm 57.49$ & \multirow{2}{*}{$G \times P: 0.972$} \\
\hline & Control & $501.67 \pm 57.70$ & $504.63 \pm 58.44$ & \\
\hline \multirow{2}{*}{$\mathrm{V} / \mathrm{S}$} & Exercise & $0.47 \pm 0.02$ & $0.42 \pm 0.02^{* *}$ & \multirow{2}{*}{$G \times P: 29.894^{\# \#}$} \\
\hline & Control & $0.47 \pm 0.02$ & $0.49 \pm 0.02$ & \\
\hline
\end{tabular}

$\mathrm{G} \times \mathrm{P}$ : group $\times$ period.

Significantly different from baseline: ${ }^{* *} p<0.01$.

Significant interaction among groups and period: ${ }^{\# \#} p<0.01,{ }^{\# \#} p<0.001$.

and low-density lipoprotein cholesterol (LDL-C) were analyzed enzymatically using an autoanalyzer (Hitachi 7600110/7170 Analyzer, Tokyo, Japan). Levels of TNF- $\alpha$, CD14, and immunoglobulins (IgA, IgG, and IgM) were analyzed using enzyme-linked immunosorbent assay (ELISA), flow cytometry, and immunoturbidimetry, respectively.

2.3. Data Processing. The SPSS Statistics 18.0 program was used to calculate means and standard deviations for all variables. Significant differences between the exercise and control groups were determined with 2-way ANOVA and paired $t$-tests. For independent correlation between changes in endotoxin and oxygen intake per weight, the correlation coefficient and regression coefficient of each were obtained. $p<0.05$ was considered significant.

\section{Results}

3.1. Changes in Abdominal Fat. Body composition and abdominal fat before and after the exercise program are given in Table 3 . In the exercise group, visceral fat and the visceral to subcutaneous fat ratio (both $p<0.01$ ) decreased significantly compared to preprogram values. Visceral fat $(F=7.619, p<0.01)$ and the visceral to subcutaneous fat ratio $(F=29.894, p<0.001)$ changed significantly over time and between groups. No control group variables changed after the program.

3.2. Changes in Health-Related Fitness. Health-related fitness changes are given in Table 4 . In the exercise group, a significant decrease was seen in postprogram \% body fat $(p<$ $0.05)$ compared to preprogram values. Grip strength $(p<$ $0.05)$, push-ups $(p<0.05)$, and maximum oxygen intake per weight $(p<0.01)$ increased significantly. Moreover, \% body fat $(F=4.844, p<0.05)$, grip strength $(F=4.583, p<$ $0.05)$, push-ups $(F=5.562, p<0.05)$, and maximum oxygen intake per weight $(F=7.555, p<0.01)$ changed significantly over time and between group. No control group variables changed after the program.

3.3. Changes in Serum Lipids, TNF- $\alpha$, Immune Functions, CD14, and Endotoxin. Cholesterol, TNF- $\alpha$, and C-reactive protein (CRP) level changes are given in Table 5. In the exercise group, HDL-C level increased significantly following the 12-week program $(p<0.05)$, whereas TNF- $\alpha$ level decreased significantly $(p<0.05)$. Moreover, HDL-C $(F=$ 9.728, $p<0.01)$ and TNF- $\alpha(F=5.147, p<0.05)$ 
TABLE 4: The changes of health-related fitness between the groups at baseline and after 12 weeks of training.

\begin{tabular}{|c|c|c|c|c|}
\hline Variables & Group & Baseline & 12 weeks & $F$-value \\
\hline \multirow{2}{*}{ Body weight (kg) } & Exercise & $64.9 \pm 3.83$ & $62.5 \pm 2.94$ & \multirow{2}{*}{$\mathrm{G} \times \mathrm{P}: 2.112$} \\
\hline & Control & $64.7 \pm 3.26$ & $65.3 \pm 3.36$ & \\
\hline \multirow{2}{*}{$\%$ Body fat (\%) } & Exercise & $34.3 \pm 2.78$ & $31.4 \pm 2.57^{*}$ & \multirow{2}{*}{$\mathrm{G} \times \mathrm{P}: 4.844^{\#}$} \\
\hline & Control & $34.6 \pm 1.59$ & $34.8 \pm 1.69$ & \\
\hline \multirow{2}{*}{ Lean body mass (kg) } & Exercise & $41.0 \pm 2.83$ & $42.9 \pm 2.47$ & \multirow{2}{*}{$\mathrm{G} \times \mathrm{P}: 1.502$} \\
\hline & Control & $41.2 \pm 3.53$ & $40.7 \pm 3.42$ & \\
\hline \multirow{2}{*}{ Grip strength (kg) } & Exercise & $23.88 \pm 2.97$ & $27.40 \pm 2.791^{*}$ & \multirow{2}{*}{$G \times P: 4.583$} \\
\hline & Control & $23.81 \pm 3.21$ & $23.35 \pm 2.75$ & \\
\hline \multirow{2}{*}{ Back strength (kg) } & Exercise & $49.38 \pm 3.50$ & $55.64 \pm 10.96^{*}$ & \multirow{2}{*}{$G \times P: 1.000$} \\
\hline & Control & $47.16 \pm 11.61$ & $45.85 \pm 11.16$ & \\
\hline \multirow{2}{*}{ Sit-up (frequency/30 sec) } & Exercise & $10.30 \pm 5.51$ & $13.60 \pm 3.97$ & \multirow{2}{*}{$\mathrm{G} \times \mathrm{P}: 1.740$} \\
\hline & Control & $9.00 \pm 3.94$ & $8.50 \pm 4.60$ & \\
\hline \multirow{2}{*}{ Push-up (frequency/30 sec) } & Exercise & $7.50 \pm 2.75$ & $11.90 \pm 3.66^{*}$ & \multirow{2}{*}{$G \times P: 5.562^{\#}$} \\
\hline & Control & $7.50 \pm 3.37$ & $7.10 \pm 2.99$ & \\
\hline \multirow{2}{*}{ Sit-and-reach $(\mathrm{cm})$} & Exercise & $12.21 \pm 5.26$ & $13.27 \pm 5.33$ & \multirow{2}{*}{$G \times P: 0.198$} \\
\hline & Control & $11.13 \pm 4.17$ & $10.86 \pm 3.98$ & \\
\hline \multirow{2}{*}{ Closed-eyes one-legged standing time (sec) } & Exercise & $9.22 \pm 5.82$ & $9.58 \pm 3.75$ & \multirow{2}{*}{$\mathrm{G} \times \mathrm{P}: 0.908$} \\
\hline & Control & $8.47 \pm 5.95$ & $8.37 \pm 10.82$ & \\
\hline \multirow{2}{*}{$\mathrm{VO}_{2} \max (\mathrm{mL} / \mathrm{kg} / \mathrm{min})$} & Exercise & $27.16 \pm 3.40$ & $31.75 \pm 3.87^{* *}$ & \multirow{2}{*}{$\mathrm{G} \times \mathrm{P}: 7.555^{\# \#}$} \\
\hline & Control & $27.67 \pm 3.68$ & $26.89 \pm 3.37$ & \\
\hline
\end{tabular}

$\mathrm{G} \times \mathrm{P}$ : group $\times$ period.

Significantly different from baseline: ${ }^{*} p<0.05,{ }^{* *} p<0.01$.

Significant interaction among groups and period: ${ }^{\#} p<0.05,{ }^{\# \#} p<0.01$.

TABLE 5: The changes of serum lipids and TNF- $\alpha$ between the groups at baseline and after 12 weeks.

\begin{tabular}{|c|c|c|c|c|}
\hline Variables & Group & Baseline & 12 weeks & $F$-value \\
\hline \multirow{2}{*}{ HDL-C (mg/dL) } & Exercise & $50.97 \pm 3.86$ & $55.74 \pm 2.33^{* *}$ & \multirow{2}{*}{$G \times P: 9.728^{\# \#}$} \\
\hline & Control & $50.98 \pm 3.53$ & $49.10 \pm 3.54$ & \\
\hline \multirow{2}{*}{ LDL-C (mg/dL) } & Exercise & $148.20 \pm 25.66$ & $135.10 \pm 16.96$ & \multirow{2}{*}{$G \times P: 1.121$} \\
\hline & Control & $143.80 \pm 28.73$ & $146.60 \pm 21.99$ & \\
\hline \multirow{2}{*}{$\mathrm{TC}(\mathrm{mg} / \mathrm{dL})$} & Exercise & $219.40 \pm 36.73$ & $192.30 \pm 34.72$ & \multirow{2}{*}{$G \times P: 1.566$} \\
\hline & Control & $216.50 \pm 36.50$ & $218.60 \pm 39.46$ & \\
\hline \multirow{2}{*}{$\mathrm{TG}(\mathrm{mg} / \mathrm{dL})$} & Exercise & $137.10 \pm 16.07$ & $125.00 \pm 11.03$ & \multirow{2}{*}{$G \times P: 2.863$} \\
\hline & Control & $135.50 \pm 15.93$ & $138.80 \pm 13.93$ & \\
\hline \multirow{2}{*}{ TNF- $\alpha(\mathrm{pg} / \mathrm{mL})$} & Exercise & $2.32 \pm 0.50$ & $1.90 \pm 0.05^{*}$ & \multirow{2}{*}{$\mathrm{G} \times \mathrm{P}: 5.147^{\#}$} \\
\hline & Control & $2.32 \pm 0.25$ & $2.42 \pm 0.24$ & \\
\hline
\end{tabular}

G $\times$ P: group $\times$ period.

Significantly different from baseline: ${ }^{*} p<0.05,{ }^{* *} p<0.01$.

Significant interaction among groups and period: ${ }^{\#} p<0.05,{ }^{\# \#} p<0.01$.

HDL: high-density lipoprotein cholesterol, LDL: low-density lipoprotein cholesterol, TC: total cholesterol, TG: triglyceride, and TNF- $\alpha$ : tumor necrosis factor$\alpha$.

levels changed significantly over time and between groups. No control group variables changed after the program.

Immune functions, CD14, and endotoxin values from before and after the program are given in Table 6 . Values of $\operatorname{IgA}(p<0.01)$ and $\operatorname{IgG}(p<0.05)$ increased significantly after program, whereas CD14 $(p<0.05)$ and endotoxins $(p<0.01)$ decreased significantly. Moreover, $\operatorname{IgA}(F=8.333$, $p<0.01)$, CD14 $(F=5.434, p<0.05)$, and endotoxin $(F=$ $8.963, p<0.01)$ levels changed significantly over time and between groups. No control group variables changed after the program.

\section{Discussion}

4.1. Effects on Abdominal Fat. Postmenopausal women often develop abdominal obesity without weight change from excessive accumulation of visceral fat in the abdomen [3], attributed to decreased lipolysis as a result of reduced estrogen [22]. As a result, many postmenopausal women experience decreased LBM and increased \% body fat, most frequently in the abdominal area [23]. Postmenopausal women are also more at risk from obesity than premenopausal women [24]. That is, more severe abdominal obesity results 
TABLE 6: The changes of immune function and CD14 and endotoxin between the groups at baseline and after 12 weeks.

\begin{tabular}{|c|c|c|c|c|}
\hline Variables & Group & Baseline & 12 weeks & $F$-value \\
\hline \multirow{2}{*}{$\operatorname{IgA}(\mathrm{mg} / \mathrm{dL})$} & Exercise & $211.70 \pm 18.13$ & $256.50 \pm 48.75^{* *}$ & \multirow{2}{*}{$G \times P: 8.333^{\# \#}$} \\
\hline & Control & $217.80 \pm 34.24$ & $200.80 \pm 26.55$ & \\
\hline \multirow{2}{*}{$\operatorname{IgG}(\mathrm{mg} / \mathrm{dL})$} & Exercise & $1365.10 \pm 83.46$ & $1376.80 \pm 70.30$ & \multirow{2}{*}{$G \times P: 0.791$} \\
\hline & Control & $1353.40 \pm 89.20$ & $1320.00 \pm 76.47$ & \\
\hline \multirow{2}{*}{$\operatorname{IgM}(\mathrm{mg} / \mathrm{dL})$} & Exercise & $109.35 \pm 20.98$ & $112.78 \pm 22.00$ & \multirow{2}{*}{$\mathrm{G} \times \mathrm{P}: 0.542$} \\
\hline & Control & $109.60 \pm 17.35$ & $104.00 \pm 16.67$ & \\
\hline \multirow{2}{*}{ CD14 } & Exercise & $5.84 \pm 0.67$ & $5.20 \pm 0.57^{*}$ & \multirow{2}{*}{$\mathrm{G} \times \mathrm{P}: 5.434^{\#}$} \\
\hline & Control & $5.99 \pm 0.60$ & $6.18 \pm 0.34$ & \\
\hline \multirow{2}{*}{ Endotoxin (EU/mL) } & Exercise & $0.33 \pm 0.03$ & $0.29 \pm 0.03^{* *}$ & \multirow{2}{*}{$\mathrm{G} \times \mathrm{P}: 8.963^{\#}$} \\
\hline & Control & $0.33 \pm 0.03$ & $0.35 \pm 0.03$ & \\
\hline
\end{tabular}

G $\times$ P: group $\times$ period.

Significantly different from baseline: ${ }^{*} p<0.05,{ }^{* *} p<0.01$

Significant interaction among groups and period: ${ }^{\#} p<0.05,{ }^{\# \#} p<0.01$.

CD14: cluster of differentiation 14, IgA: immune globulin A, IgG: immune globulin G, and IgM: immune globulin M.

in greater probability of metabolic disease development [25]. Therefore, prevention and mitigation of abdominal obesity in middle-aged postmenopausal women are important.

Kim et al. [26] reported that combined exercise and stretching ( $80 \mathrm{~min}$ sessions 5 times a week for 8 weeks) reduced visceral fat in middle-aged obese women. Park et al. [27] reported that an aerobic exercise regimen $(60 \mathrm{~min}$ sessions 5 times a week for 12 weeks) reduced visceral fat in middle-aged men. Choi and Chun [5] also reported that circuit training (60 min sessions 4 times a week for 12 weeks) mitigated obesity.

In the present study, a 90 min combined exercise session (10 min warm-up, aerobic exercise for 40 minutes, resistance exercise for $30 \mathrm{~min}, 10 \mathrm{~min}$ cool-down) was performed 3 times a week for 12 weeks, resulting in reduced visceral fat $(p<0.01)$ and a reduced visceral to subcutaneous fat ratio $(p<0.001)$. In addition, a significant difference in results was seen between the control and exercise groups and over time, consistent with previous studies.

These results indicate that regularly performing combined exercise helps reduce visceral and subcutaneous abdominal fat in postmenopausal women. Combined exercise is thus useful for prevention and mitigation of obesity in this group.

4.2. Changes in Health-Related Fitness. The ACSM [28] separates motor skill-related fitness from health-related fitness and divides health-related fitness into body composition, flexibility, muscle strength, muscle endurance, and cardiovascular endurance. Decline in any of these elements' scan results in an increased likelihood of contracting a disease [29].

Health-related fitness is closely related to daily life. Leaner body composition decreases the probability of obesity and metabolic disease. Greater muscle endurance increases motor performance and decreases the probability of developing hypokinetic diseases. Flexibility can reduce muscle and joint injuries, and increased cardiovascular endurance reduces the probability of developing cardiovascular diseases [30]. Clearly, health-related fitness is very important in maintaining and improving the quality of daily life, a view supported by many studies. Kim et al. [31] reported that body fat, \% body fat, and cardiorespiratory fitness improved in severely obese women as a result of a 12-week, low-intensity walking program. Shin et al. [32] reported that body fat, \% body fat, muscle mass, cardiovascular endurance, muscle strength, and flexibility improved significantly in obese female college students as a result of dumbbell lifting and walking for $60 \mathrm{~min}$ sessions 4 times a week for 12 weeks.

Although exercise type and intensity influence areas of improvement, most previous studies reported that exercise improved overall health-related fitness. This study supported those findings. The 12-week combined exercise program reduced body fat and \% body fat and improved cardiorespiratory fitness through aerobic exercise and also improved muscle strength and endurance by increasing LBM through resistance exercise. It is thus considered appropriate exercise to improve obese people's overall health-related fitness.

4.3. Changes in Serum Lipids and TNF- $\alpha$. The functions and roles of serum lipid components occur mostly in the liver. They do not exist independently in the blood but are transported combined with proteins in particle form as "lipoproteins." Lipoproteins are classified as HDL-C (transporting cholesterol from peripheral tissues to the liver for degradation) or LDL-C (accumulating cholesterol in the blood vessels) [33]. Obesity increases the prevalence of metabolic diseases, such as cardiovascular disease and insulin resistance, by raising these serum lipid levels, and HDL-C and LDL-C, in particular, are important indicators for prediction and evaluation of coronary artery diseases. Park et al. [34] reported significant increases in HDL-C and significant decreases in TC among serum lipids in middle-aged women with abdominal obesity after performing a combined exercise regimen of $60 \mathrm{~min}$ sessions 4 times a week for 12 weeks. Kang and Jung [35] also reported significant decreases in TC and significant increases in HDL-C in obese people after an aerobic exercise regimen of 40-minute sessions 4 times a week for 12 weeks.

In the present study, the exercise group's LDL-C, TC, and TG values decreased, while HDL-C values increased. 
Although Paek [36] reported that there were no significant differences in middle-aged women's TC values after performing aerobic dance 4 times a week for 12 weeks, most studies reported positive improvements in serum TC and TG levels as a result of regular training. In particular, HDL$\mathrm{C}$ improvements, a cardiovascular risk factor, were seen in the present study. We believe that combined exercise can strengthen cardiopulmonary functions and help prevent and mitigate cardiovascular diseases and hyperlipidemia from HDL-C caused obesity.

Additionally, TNF- $\alpha$, one of the inflammatory cytokines and a primary modulator of systematic response to infectious diseases, is closely related to obesity. Its serum concentrations are known to increase during high-intensity exercise, and high levels of TNF- $\alpha$ are secreted by adipose tissues [37].

Samartín and Chandra [38] reported that TNF- $\alpha$ and TNF- $\alpha$ gene activations, accompanied by weight loss, were observed in obese people. Ziccardi et al. [39] conducted a 1year weight-loss program with 56 middle-aged obese women and saw a significant TNF- $\alpha$ decrease. Kondo et al. [40] reported significant decreases in TNF- $\alpha$ in obese women after 7 months of slope jogging and walking. This study's decrease in TNF- $\alpha$ levels was consistent with previous studies and is believed to result from reduced adipose tissue secretion of TNF- $\alpha$ after combined exercise decreased body fat, $\%$ body fat, and visceral fat.

4.4. Changes in Immune Functions, CD14, and Endotoxins. Immunoglobulins (IgA, IgG, and IgM) function as antibodies, carrying out humoral immune functions against antigens that invade the body [41].

$\operatorname{IgA}$ is found mostly in saliva and stops bacteria or microorganisms from invading the body by binding to them at sites vulnerable to bacterial invasion. IgG is the most abundant serum antibody. IgM is early antibody and is highly involved with bacteriolysis. The known normal values of immunoglobulins $\operatorname{IgA}$, IgG, and $\operatorname{IgM}$ are $160-330 \mathrm{mg} / \mathrm{dL}$, 700-1400 mg/dL, and 60-150 mg/dL, respectively [42].

Nehlsen-Cannarella et al. [43] reported that appropriate exercise increased the serum immunoglobulin concentrations, whereas Mackinnon [44] found no effect. Chun et al. [45] reported that IgM decreased significantly in middleaged women following combined exercise for 12 weeks, and Tennyson and Friedman [7] reported significant increases in IgG in middle-aged obese women after 12 weeks of Nordic walking. In the present study, IgA increased significantly. In summary, based on this study and previous studies, it is believed that the immunoglobulins concentrations differ according to exercise time and duration, intensity, and participant age.

Endotoxins stimulate microphages by binding to CD14 on the microphage membrane. The stimulated microphages secrete proinflammatory cytokines, such as TNF- $\alpha$, IL-6, and IL- $1 \beta$, sometimes triggering excessive innate immune responses and causing a variety of immune responses and diseases, as well as aggravating existing diseases $[9,46]$. In addition, when concentrations of serum endotoxin exceed 2 to 3 times the normal level, it becomes difficult to modulate the extent of inflammation, which induces weight gain [47]. In other words, endotoxins are believed to be important obesity-related and metabolic disease risk factors.

While studies on endotoxins and exercise responses are lacking, the present study showed that visceral fat and endotoxin decrease after a 12-week combined exercise program. Therefore, combined exercise is likely effective in reducing endotoxin concentrations. Lowered TNF- $\alpha$ after exercise might have an important role in the obesity reduction and increased immune function.

\section{Conclusions}

In the present study, middle-aged, postmenopausal women with abdominal obesity performed 90 minutes of combined aerobic and resistance exercises 3 times a week for 12 weeks in order to examine the effects of combined exercise on healthrelated fitness, endotoxin levels, and immune functions. The following conclusions were obtained.

We conclude that, as a result of the combined exercise program, abdominal fat, health-related fitness, immune response, and endotoxins all changed significantly over time and between groups. As seen in the above results, the exercise group's abdominal obesity was mitigated due to visceral fat reduction; grip strength, push-ups, and oxygen intake per weight improved; and HDL-C and IgA increased, while TNF$\alpha, \mathrm{CD} 14$, and endotoxin levels decreased.

Therefore, for middle-aged postmenopausal women with abdominal obesity, a 12-week combined exercise regimen improved \% body fat and health-related fitness and increased immune function, while a decrease in visceral fat and endotoxin concentrations was seen. Accordingly, we believe that combined exercise is effective in mitigating abdominal obesity, preventing metabolic diseases, and enhancing immune function. Additionally, endotoxins may be a potential risk factor for obesity. Lowered TNF- $\alpha$ after exercise might have an important role in the obesity reduction and increased immune function.

In addition, making specific diet programs for postmenopausal woman to achieve a better control of variables on preventing metabolic diseases and mitigating obesity is urgently needed in the near future.

\section{Conflict of Interests}

All authors declare that there is no conflict of interests regarding the publication of this paper.

\section{References}

[1] Ministry for Health- Welfare and Family Affairs and Korea Centers for Disease Control and Prevention, Korean National Health and Nutrition Survey, 2009.

[2] R. H. Eckel, Obesity-Mechanisms and Clinical Management, Lippincott Williams \& Wilkins, Philadelphia, Pa, USA, 2003.

[3] T. Douchi, S. Yamamoto, S. Nakamura et al., "The effect of menopause on regional and total body lean mass," Maturitas, vol. 29, no. 3, pp. 247-252, 1998. 
[4] W. J. Cho, "The effect of combined exercise program on health related physical fitness and blood variables in obese middleaged women," Journal of Sport and Leisure Studies, vol. 39, pp. 645-658, 2010.

[5] O. J. Choi and S. Y. Chun, "The effects of 12 weeks circuit training on body composition and coronary artery risk factors in menopausal obese women," Journal of Sport and Leisure Studies, vol. 38, pp. 961-970, 2009.

[6] S. K. Park, Y. C. Kwon, and E. H. Kim, "Effect of the yoga program on health related fitness, depression, stress related factors and immune cell in middle-aged women," Journal of Sport and Leisure Studies, vol. 33, no. 2, pp. 999-1010, 2008.

[7] C. A. Tennyson and G. Friedman, "Microecology, obesity, and probiotics," Current Opinion in Endocrinology, Diabetes and Obesity, vol. 15, no. 5, pp. 422-427, 2008.

[8] J. H. Jung, M. K. Kang, J. S. No, and K. S. Youm, "The Association between Visceral Fat and Endotoxin," Journal of Korean Society for the Study of Obesity, vol. 19, no. 3, pp. 78-84, 2010.

[9] C. B. S. Henry and B. R. Duling, "TNF-alpha increases entry of macromolecules into luminal endothelial cell glycocalyx," The American Journal of Physiology-Heart and Circulatory Physiology, vol. 279, no. 6, pp. H2815-H2823, 2000.

[10] S. Genth-Zotz, S. von Haehling, A. P. Bolger et al., "Pathophysiologic quantities of endotoxin-induced tumor necrosis factoralpha release in whole blood from patients with chronic heart failure," American Journal of Cardiology, vol. 90, no. 11, pp. 12261230, 2002.

[11] A. B. Hauser, A. E. M. Stinghen, S. M. Gonçalves, S. Bucharles, and R. Pecoits-Filho, "A gut feeling on endotoxemia: causes and consequences in chronic kidney disease," Nephron Clinical Practice, vol. 118, no. 2, pp. c165-c172, 2011.

[12] K.-U. Belge, F. Dayyani, A. Horelt et al., “The proinflammatory $\mathrm{CD}_{14}{ }^{+} \mathrm{CD} 16^{+} \mathrm{DR}^{++}$monocytes are a major source of TNF," The Journal of Immunology, vol. 168, no. 7, pp. 3536-3542, 2002.

[13] A. Bowie and L. A. J. O’Neill, “The interleukin-1 receptor/Tolllike receptor superfamily: signal generators for proinflammatory interleukins and microbial products," Journal of Leukocyte Biology, vol. 67, no. 4, pp. 508-514, 2000.

[14] N. A. Skinner, C. M. MacIsaac, J. A. Hamilton, and K. Visvanathan, "Regulation of Toll-like receptor (TLR)2 and TLR4 on $\mathrm{CD} 14^{d i m} \mathrm{CD} 16^{+}$monocytes in response to sepsis-related antigens," Clinical and Experimental Immunology, vol. 141, no. 2, pp. 270-278, 2005.

[15] O. S. Al-Attas, N. M. Al-Daghri, K. Al-Rubeaan et al., "Changes in endotoxin levels in T2DM subjects on anti-diabetic therapies," Cardiovascular Diabetology, vol. 8, article 20, 2009.

[16] L. Chávez-Sánchez, K. Chávez-Rueda, M. V. Legorreta-Haquet et al., "The activation of CD14, TLR4, and TLR2 by MmLDL induces IL- $1 \beta$, IL- 6 , and IL-10 secretion in human monocytes and macrophages," Lipids in Health and Disease, vol. 9, article 117, 2010.

[17] N. L. Kim, H. Y. Park, and S. N. Nam, "Change of cellular immunity substance by exercise types in elderly women," Korean Journal of Societyof Growth \& Development, vol. 18, no. 3, pp. 163-170, 2010.

[18] Q. Y. Ng, K. W. Lee, C. Byrne, T. F. Ho, and C. L. Lim, "Plasma endotoxin and immune responses during a $21-\mathrm{km}$ road race under a warm and humid environment," Annals of the Academy of Medicine Singapore, vol. 37, no. 4, pp. 307-314, 2008.
[19] R. P. Sloan, P. A. Shapiro, R. E. DeMeersman et al., "Aerobic exercise attenuates inducible TNF production in humans," Journal of Applied Physiology, vol. 103, no. 3, pp. 1007-1011, 2007.

[20] ACSM, ACSM's Guidelines for Exercise Testing and Prescription, 2006.

[21] Korea Sport Science Institute, Sport White Paper, 2010.

[22] M. Gambacciani and A. Pepe, "Menopause and related problems," Minerva Medica, vol. 98, no. 3, pp. 191-201, 2007.

[23] C. J. Ley, B. Lees, and J. C. Stevenson, "Sex and menopauseassociated changes in body-fat distribution," The American Journal of Clinical Nutrition, vol. 55, no. 5, pp. 950-954, 1992.

[24] S. A. Shin and H. S. Kang, "The purpose of this study was to compare obesity indices, cardiorespiratory fitness, metabolic syndrome indices between pre-and post-menopausal women," Journal of Exercise Science, vol. 14, no. 2, pp. 193-202, 2005.

[25] J. H. Lee, I. K. Paek, H. S. Kim, B. S. Cha, H. C. Lee, and K. P. Heo, "Body fat distribution and serum levels of lipids and hormones in pre- and post-menopausal wonen," Journal of Lipidology and Atherosclerosis, vol. 7, no. 2, pp. 250-260, 1997.

[26] J. H. Kim, K. P. Park, and W. J. Lee, "An experimental research on the effect of complex exercise which combined with stretching program on body composition, visceral fat and adipocytokines in elderly obese women," Journal of Sport and Leisure Studies, vol. 41, pp. 729-742, 2010.

[27] S. K. Park, Y. C. Kwon, and J. K. Park, "Effect of aerobic exercise on carotid artery structure, function and vascular endothelial growth factor (VEGF) in abdominal obese men," The Korean Journal of Physical Education, vol. 47, no. 3, pp. 473-484, 2008.

[28] American College of Sports Medicine, ACSM's Guidelines for Exercise Testing and Rescription, 8th edition, 2010.

[29] C. B. Corbin and R. Lindsey, Concepts of Physical Fitness with Laboratories, Brown and Benchmark, 2001.

[30] S. J. Kang and Y. D. Won, Coaching, Dae Media, Seoul, Republic of Korea, 1994.

[31] K. J. Kim, S. Y. Park, J. H. Lee, J. M. Jung, H. S. No, and H. M. Choi, "The effect of low intensity exercise for physical fitness body composition and blood lipids in obese women," Korean Journal of Society of Growth \& Development, vol. 18, no. 1, pp. 19-24, 2010.

[32] D. S. Shin, C. J. Lee, and D. J. No, "The Effect of walking and walking with dumbbell exercise on health-related fitness and blood Lipids in obese female college students," Journal of Korean Society for the Study of Physical Education, vol. 15, no. 2, pp. 141$159,2010$.

[33] S. M. Grundy, "Obesity, metabolic syndrome, and cardiovascular disease," The Journal of Clinical Endocrinology and Metabolism, vol. 89, no. 6, pp. 2595-2600, 2004.

[34] S. Park, Y. C. Kwon, E. H. Kim, and J. H. Jang, "The effects of combined training on visceral fat, adiponectin and TNF- $\alpha$ concentration in middle aged abdominal obese women," Korean Journal of Societyof Growth \& Development, vol. 14, no. 4, pp. 63-77, 2006.

[35] S. J. Kang and S. L. Jung, "Effect of 12 weeks aerobic exercise on obesity index, insulin resistance, cardiovascular disease risk factors and exercise capacity in obese adolescent," Journal of Exercise science, vol. 19, no. 3, pp. 277-288, 2010.

[36] W. D. Paek, Effects of Aerobic Dance on the Blood Lipid, Functional Physical Ability of Middle-Aged Woman, Josun University Sport Science Institute, 1993.

[37] G. S. Hotamisligil and B. M. Spiegelman, "Tumor necrosis factor $\alpha$ : a key component of the obesity-diabetes link," Diabetes, vol. 43, no. 11, pp. 1271-1278, 1994. 
[38] S. Samartín and R. K. Chandra, "Obesity, overnutrition and the immune system," Nutrition Research, vol. 21, no. 1-2, pp. 243262, 2001.

[39] P. Ziccardi, F. Nappo, G. Giugliano et al., "Reduction of inflammatory cytokine concentrations and improvement of Endothelial functions in obese women after weight loss over one year," Circulation, vol. 105, no. 7, pp. 804-809, 2002.

[40] T. Kondo, I. Kobayashi, and M. Murakami, "Effect of exercise on circulating adipokine levels in obese young women," Endocrine Journal, vol. 53, no. 2, pp. 189-195, 2006.

[41] W. S. Eom, Effect of chronic aerobic exercise with different exercise intensity on VO2max, MDA, SOD, immune function [Ph.D. thesis], Seoul University Graduate School, 2004.

[42] G. N. Lee and O. H. Kwon, Clinical Pathology File, Medicine Paradigm of Culture Group, Seoul, Republic of Korea, 2003.

[43] S. L. Nehlsen-Cannarella, D. C. Nieman, J. Jessen et al., "The effects of acute moderate exercise on lymphocyte function and serum immunoglobulin levels," International Journal of Sports Medicine, vol. 12, no. 4, pp. 391-398, 1991.

[44] L. T. Mackinnon, "Chronic exercise training effects on immune function," Medicine \& Science in Sports \& Exercise, vol. 32, no. 7, pp. S369-S376, 2000.

[45] T. W. Chun, K. S. Park, and D. I. Seo, "Effects of dombined exercise training on endocrine system and immunoglobulin in middle aged women," Exercise Science, vol. 17, no. 2, pp. 157-162, 2008.

[46] W. Pruzanski, P. Vadas, and J. Browning, "Secretory nonpancreatic group II phospholipase A2: role in physiologic and inflammatory processes," Journal of Lipid Mediators, vol. 8, no. 3, pp. 161-167, 1993.

[47] P. D. Cani, J. Amar, M. A. Iglesias et al., "Metabolic endotoxemia initiates obesity and insulin resistance," Diabetes, vol. 56, no. 7, pp. 1761-1772, 2007. 


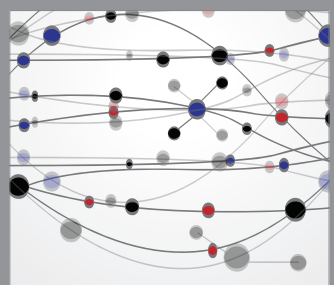

The Scientific World Journal
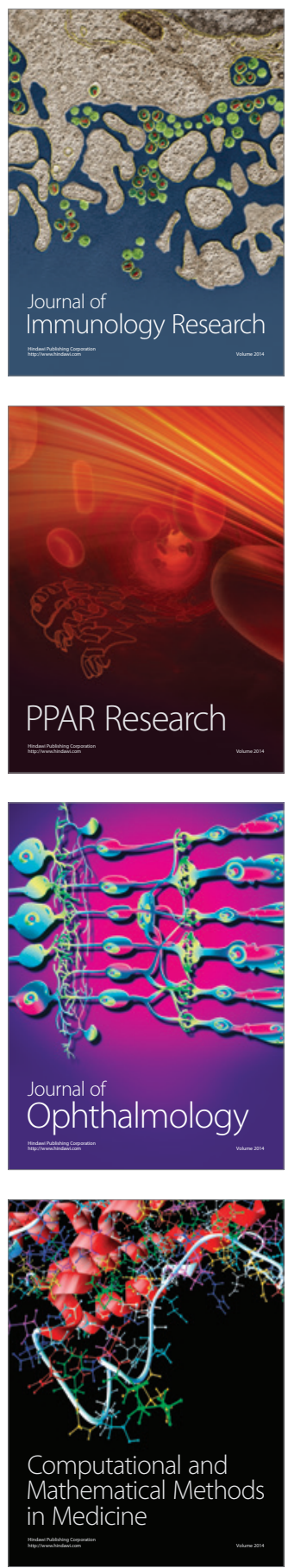

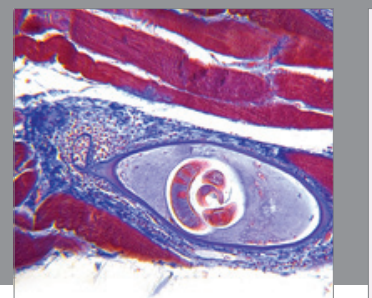

Gastroenterology

Research and Practice
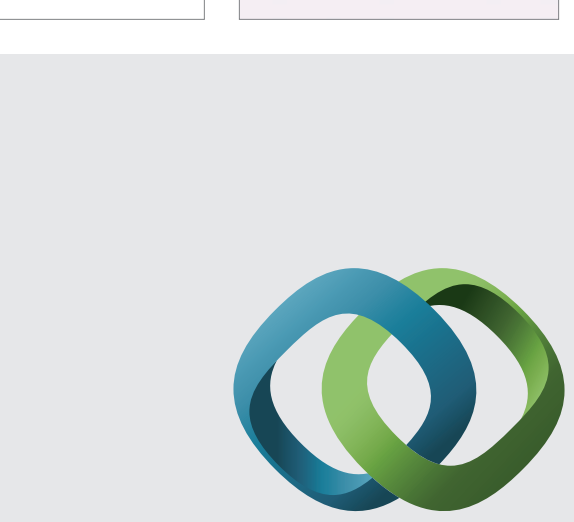

\section{Hindawi}

Submit your manuscripts at

http://www.hindawi.com
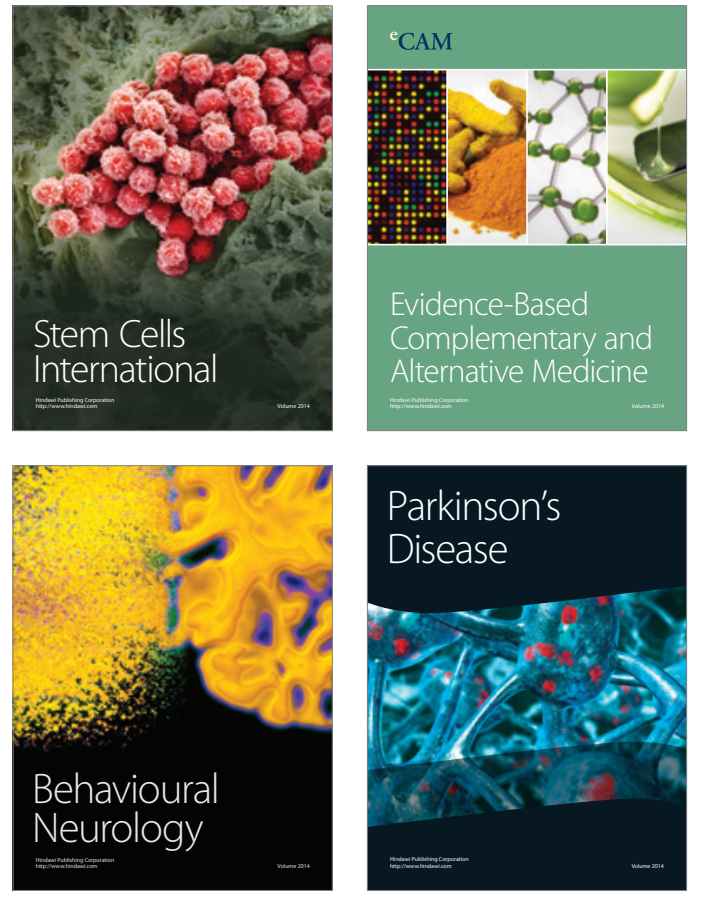
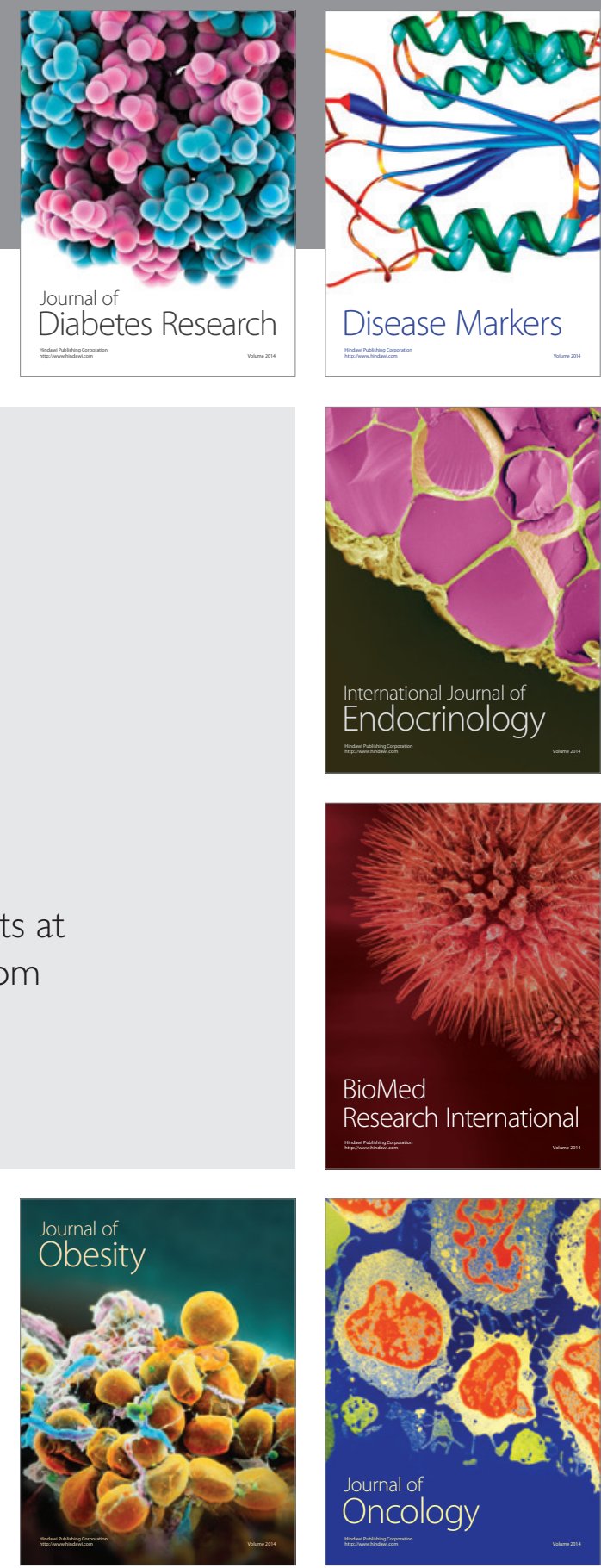

Disease Markers
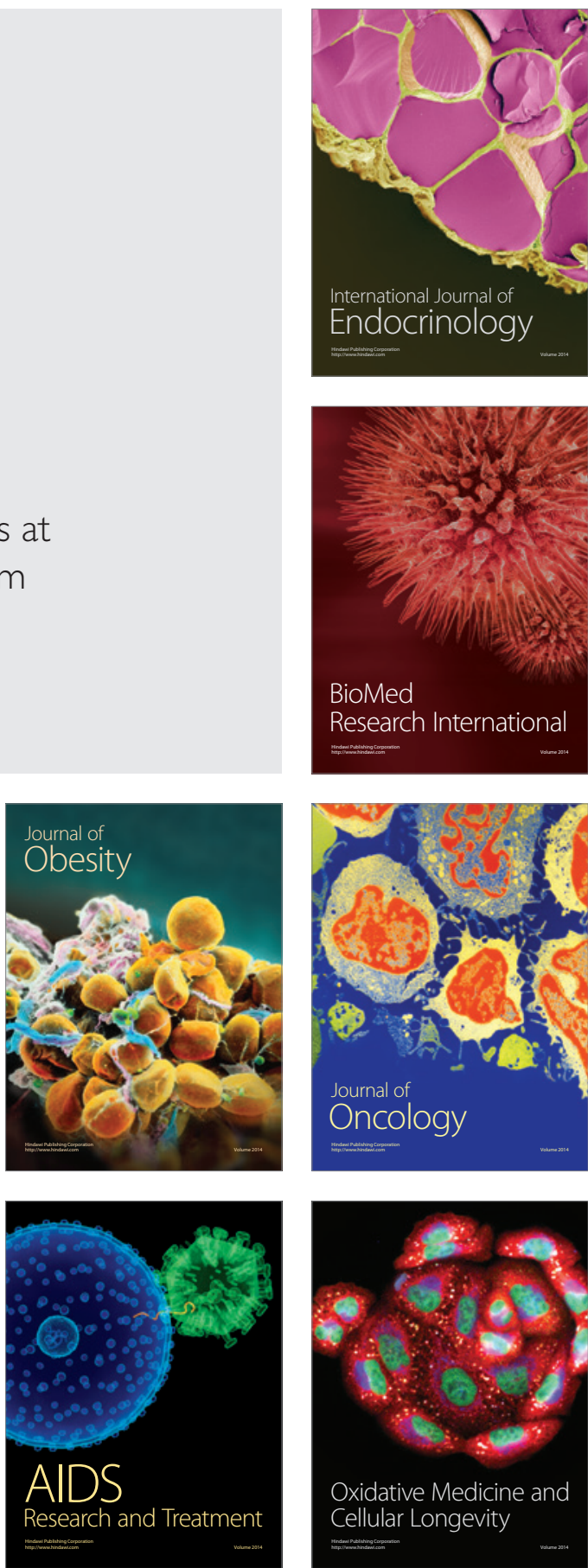